\title{
Sperm capacitation and motility could induce the chromosomal anomaly of bovine embryo
}

\section{Editorial}

We would like to introduce my research article which is "Changes in Sperm Motility and Capacitation Induce Chromosomal Aberration of the Bovine Embryo following Intracytoplasmic Sperm Injection" as a Research Article.

Intracytoplasmic sperm injection (ICSI) has become the method of choice with which to treat human male infertility. Birth and implantation rates following human ICSI are known to be lower than with in vitro fertilization. One of the largest outstanding problems associated with this technique is our current lack of knowledge concerning the effect of sperm capacitation and motility upon the subsequent development of injected oocytes. We have previously demonstrated that the methyl- $\beta$-cyclodextrin induced capacitation of sperm promoted fertilization in vitro. ${ }^{1}$ We showed that single activated sperm caught could be caught using a micropipette and stained with chlortetracycline after sperm were cultured in media containing methyl-b-cyclodextrin. Following capture, individual activated sperm mostly exhibited a pattern characteristic of capacitation. ${ }^{2}$ However, the effect of capacitated sperm and activated motile sperm upon embryo development following ICSI remains less well defined.

Capacitated sperm are known to produce reactive oxygen species, such as hydrogen peroxide and superoxide. Motility activation involves increased sperm tail movement and is an energetically intensive process. Mitochondria are known to produce ROS as well as ATP in motile sperm. However, ROS are also able to diffuse and pass through cell membranes and can influence most types of cellular molecules such as lipids, protein and nucleic acids. There is a possibility therefore, that ROS produced by capacitated and activated sperm affect embryogenesis following ICSI in human and animal oocytes. ${ }^{3}$ In human ICSI, the normal method is that progressive motility sperm is selected and injected into oocytes. There is a possibility that the selection of progressive motile sperm is a potentially dangerous factor for early embryo development an $\mathrm{d}$ fetus growth following human ICSI.

The present study showed that the selection and injection of activated sperm did not affect early embryo development but induced chromosomal aberration at the blastocyst stage. Furthermore, ROS produced by the mitochondria of capacitated, activated sperm induced chromosomal aberration at the blastocyst stage following ICSI. It remains a possibility that ROS produced by sperm mitochondria is a

\author{
Volume 3 Issue I - 2015 \\ Yoku Kato \\ Department of Infertility, Royal Bell Clinic, Japan
}

Correspondence: Yoku Kato, Department of Infertility, Royal Bell Clinic, Narumi-cho Mizuhiroge 93-195, Midori-ku, Nagoya, Aichi, 458-080I, Japan, Tel 8I-052-879-6673,

Email yoku-kato@kishokai.or.jp

Received: October 25, 2015 | Published: October 26, 2015

potentially dangerous factor when ICSI is carried out in humans and domestic animals. When ICSI is used for the in vitro production of offspring, the injection of normal motile sperm or activated sperm in which the mitochondria have been inhibited could improve the quality of ICSI-derived embryos. The present study suggests that the selective injection of sperm producing less ROS may contribute to safer growth of human embryos following ICSI. We suggested that this work is of great interest and the benefit of introduction for my editorial to a broad audience in this Obstetrics \& Gynecology International Journal.

\section{Acknowledgments}

None.

\section{Conflicts of interest}

The authors declare there is no conflict of interests.

\section{References}

1. Nagao Y, Harada Y, Yamaguchi M, et al. Antioxidant treatment during preservation of bovine ovaries increased the development potential of embryos. Zygote. 2010;18(4):315-321.

2. Kato Y, Nagao Y. Capacitation status of activated bovine sperm cultured in media containing methyl-beta-cyclodextrin affects the acrosome reaction and fertility. Zygote. 2010;19(1):21-30.

3. Kato Y, Nagao Y. Changes in sperm motility and capacitation induce chromosomal aberration of the bovine embryo following intracytoplasmic sperm injection. PLoS ONE. 2015;10(6):e0129285. 